\title{
Comparative Performance Analysis of Secured LWT- SVD Based Color Image Watermarking Technique in YUV, YIQ and YCbCr Color Spaces
}

\author{
Shveti Sejpal \\ Research Scholar \\ Saurashtra University \\ Rajkot, Gujarat, India.
}

\author{
Nikesh Shah, PhD \\ Professor \\ Department of Physics, Saurashtra University \\ Rajkot, Gujarat, India.
}

\begin{abstract}
In this paper the perceptual quality and robustness in LWT domain and DWT domain is evaluated for the gray-scale image. The proposed technique is based on Lifting Wavelet Transform and Discrete Wavelet Transform (LWT-SVD) domain for embedding the gray-scale image to the color host image. Fibonacci-Lucas Transform is used to scramble the watermark image for security reason. YUV, YIQ and $\mathrm{YCbCr}$ color spaces are evaluated by implementing this secured robust algorithm. $\mathrm{Y}, \mathrm{U}, \mathrm{V}, \mathrm{I}, \mathrm{Q}, \mathrm{Cb}$ and $\mathrm{Cr}$ channels are examined for the perceptual quality and robustness. Maximum recorded PSNR for V channel is 90.6039 and up to 65.5971 for $\mathrm{Y}$ channel in $\mathrm{YCbCr}$ color space. Maximum $\mathrm{NC}$ is recorded in $\mathrm{Y}$ channel for all the color spaces. Applied various attacks like Average filter, Gaussian filter, Gaussian Noise, Salt \& pepper noise, Blur, Motion Blur, Crop, JPEG Compress, Rotate, etc. to the proposed technique to test all the channels of the different color spaces.
\end{abstract}

\section{General Terms}

Color Image, Digital Watermarking, Information Hiding, Robustness, Security.

\section{Keywords}

Color Image Watermarking, Discrete Wavelet Transform, Lifting Wavelet Transform, Fibonacci Lucas Transform.

\section{INTRODUCTION}

Digital watermark determines the copyrighted from noncopyrighted content files within this distributed system architecture. With the use of digital watermarks the copyrights and usage rights of digital content can be communicated by embedding the content identifiers in content. Watermarks can be embedded in spatial domain and frequency domain. In spatial domain it can be fragile. Digital color image watermarking can be implemented in different color spaces like RGB, YUV, YIQ, YCbCr, etc. Paper is organized into different section as related work, Foundation of proposed Method, proposed method, experimental result, and conclusion.

\section{RELATED WORK}

These are some existing color watermarking technique in wavelet transform. H. Shi et al. [1] have proposed a color watermarking scheme in SVD domain. In this scheme they have decomposed both the host and watermark image in R, G and $\mathrm{B}$ components and performed blocking on each component and singular values are used for watermark embedding. P. Ramana [2] Reddy et al. have proposed a blind color watermarking technique in which frequency coefficients are modified to embed the watermark bits by spreading the spectrum using pseudo random sequences. A robust color watermarking scheme proposed by Baisa L. Gunjal [3]. In this technique 3-level DWT is applied on V component of YUV color space and middle frequency is used to embed the Arnold scrambled watermark image. D.Vaishnavi and T.S.Subashini [4] have proposed a color watermarking scheme in SVD domain on blue channel of RGB color space. Singular value are used to embed the watermark image. A. Tun and Y. Thein have proposed a watermarking technique in hybrid domain LWT and DCT. Here ch sub-band is used to embed the watermark. N.Venkatram et al. [5] have proposed a gray scale watermarking scheme in LWT and SVD domain. Here all the sub-band of LWT is used and SVD is applied on all the subband. The singular values are used for watermark embedding technique.

\section{FOUNDATION OF PROPOSED WORK}

\subsection{Lifting Wavelet Transform}

Signal transform is used to transform the signal to the different domain, perform several operations on the transformed signal and inverse the transform and bake to the original domain i.e. transform should be invertible. Wavelet transform can be performed using filter bank i.e. Low pass filter and high pass filter. Lifting scheme splits the samples into odd and even samples. In 1998, Sweldens et al. designed the Lifting Wavelet Transform, second generation wavelet transform to extend the utility of wavelet methods. Lifting method is simple and efficient scheme as there is no complex mathematical calculation. Digital signal are generally a sequence of integer numbers, whereas wavelet transforms outputs in floating point numbers. For the efficient inverse transformation integer to integer conversion is important. LWT can be modified to operate on integers and thus during inverse transform rounding error can be avoided.

Properties of LWT:

- Easy inverse transform is always true.

- LWT can be done in-place.

- LWT cuts the computational complexity in half compared to DWT.

LWT consist of main three steps:

1. Split: Here it splits the signal into odd signal subsets (the wavelet coefficients filtered through high-pass filter) and even signal subsets (filtered through lowpass filtered).

2. Predict: Here only even signal subsets are used to divide the signal into odd and even signal subsets by 
transforming odd signal subsets into wavelet coefficient.

3. Update: Here even signal subsets will be processed using wavelet coefficient, computed in predict phase for calculating the scaling function.

In Lifting wavelet decomposition image is decomposed in to approximation coefficient subset $(\mathrm{cA})$ and detail coefficient subsets, horizontal $(\mathrm{cH})$, vertical $(\mathrm{cV})$ and diagonal $(\mathrm{cD})$.

\subsection{Discrete Wavelet Transformation}

Discrete wavelet transform is a hierarchical transform. Through DWT signals can be analyzed at different level called as multi resolution analysis [6]. Unlike DCT, in DWT transformation is applied on whole image, not on nonoverlapped 2-D blocks. In DWT, it splits the signals by passing it through the high pass filter and low pass filter. The signal is split in to high and low frequency sub-bands. High frequency sub-band consist of edge information and human eyes are less sensitive to the changes on the edges. Applying DWT to the digital image results in 4 non-overlapping subbands, approximation sub-band $\mathrm{cA}$ and detail sub-bands $\mathrm{cH}$, $\mathrm{cV}, \mathrm{cD}$. Adding watermark to approximation sub-band affects the perceptual quality. Human Visual System is less sensitive to horizontal detail coefficient hence $\mathrm{cH}$ is selected to embed the watermark in the proposed work. There are various types of wavelet function like Daubechies, Morlet Coiflet, Marr, Harr, etc.

\subsection{Selection of Wavelet Transform \\ Domain}

Various tests are carried out for evaluating the perceptual quality and robustness in LWT domain and DWT domain for image watermarking. The cover image of size 512 x 512 and watermark image of size $256 \times 256$ is used for the test. Here the cover image was decomposed with one level 2D-DWT and 2D-LWT using Haar wavelet and the watermark is embedded in $\mathrm{cH}$ sub-band using equation (1). Here $\mathrm{K}$ is the scaling factor. And the noisy image is composed using cA, newcH, $\mathrm{cV}$ and $\mathrm{cD}$ sub-bands.

$$
\text { newcH }=c H+K * \text { Watermark }
$$

The PSNR and NC is calculated for the peppers, Lena, Baboon and Avion images. In Fig. 1 and Fig. 2, it is found that watermark embedding in LWT domain gives better perceptual quality and robustness compared to DWT domain, and thus LWT domain is used for further comparative performance analysis of various color spaces in color image watermarking scheme.

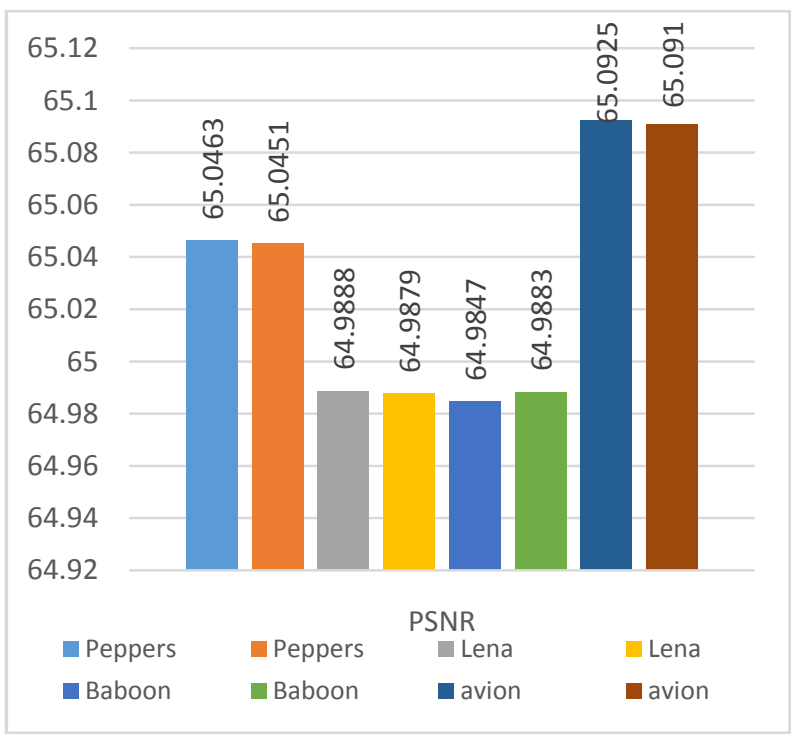

Fig 1: PSNR value for different images in DWT and LWT domain

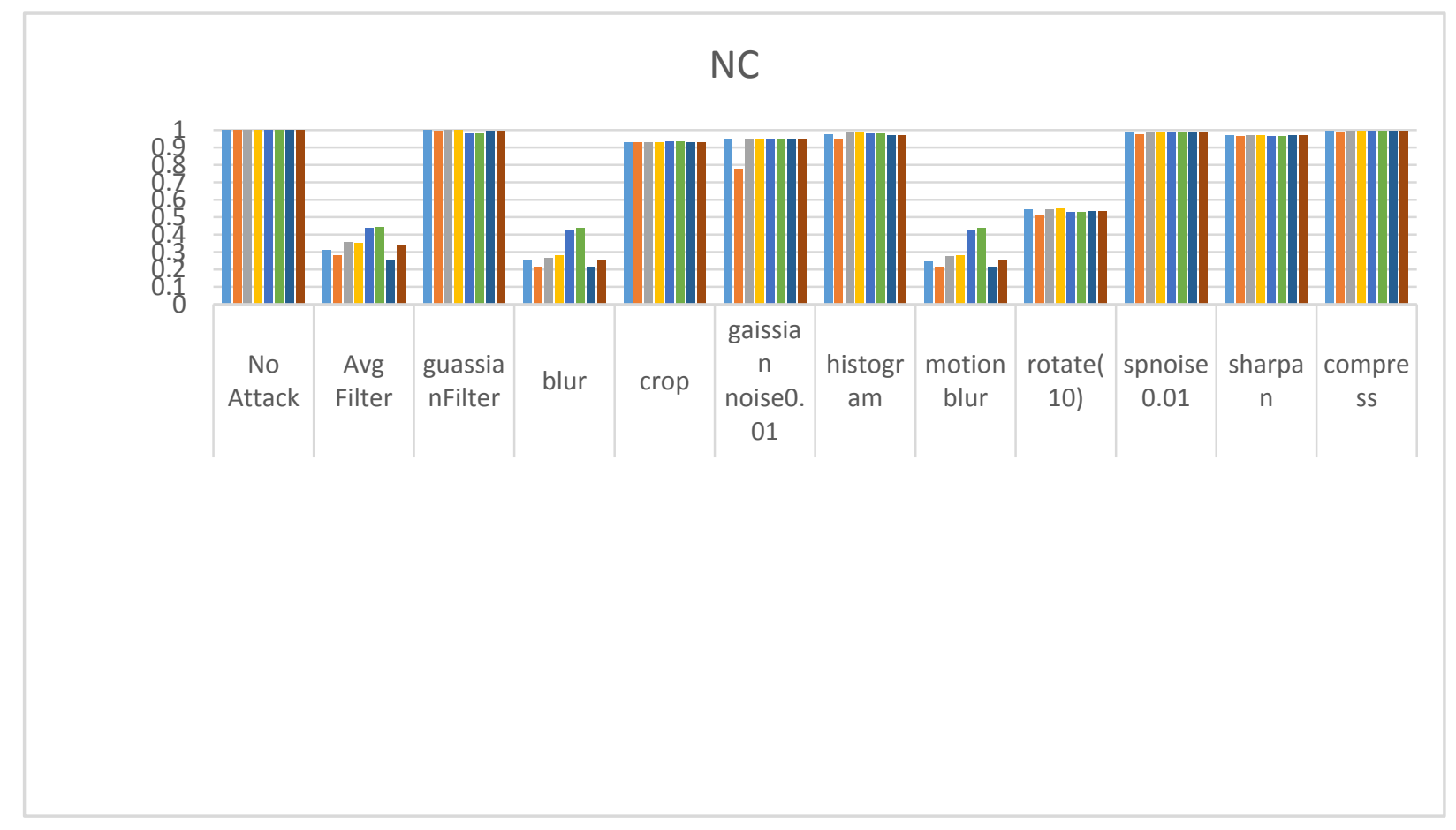

Fig 2: NC value for different images in DWT and LWT domain for different attacks 


\subsection{Singular Value Decomposition}

$\mathrm{SVD}$ is the factorization of a rectangular matrix $\mathrm{Mm} \mathrm{x} \mathrm{n}$ in to three matrices $\mathrm{U}, \mathrm{S}$ and $\mathrm{V}$. Here $\mathrm{U}$ and $\mathrm{V}$ are the orthogonal matrices and the diagonal matrix $S$ is the eigenvalues of matrix M. here $U$ is of size $m \times m$ and $V$ is of size $n \times n$. matrix $\mathrm{U}$ and $\mathrm{V}$ are also called as left and right vector of $\mathrm{M}$ respectively. The diagonal values of $\mathrm{S}$ are called as singular values of matrix $M$. Inverse SVD can be performed by multiplying these three matrices such that M=USV'. Singular values give valuable information.

For example

$$
\begin{gathered}
M=\left[\begin{array}{ll}
11 & 2 \\
22 & 4
\end{array}\right]= \\
{\left[\begin{array}{cc}
-0.4472 & -0.8944 \\
-0.8944 & 0.4472
\end{array}\right]\left[\begin{array}{cc}
25.0000 & 0 \\
0 & 0
\end{array}\right]\left[\begin{array}{cc}
-0.9839 & -0.1789 \\
-0.1789 & 0.9839
\end{array}\right]}
\end{gathered}
$$

\subsection{Color Spaces}

\subsubsection{RGB Color space}

Many researchers have used RGB color space for embedding watermark. Color image can be decomposed into Red, Green, and Blue channels using equation (2), (3) and (4)

$\mathrm{R}=$ image(:,:,1);

$\mathrm{G}=$ image(:,:,2);

$\mathrm{B}=$ image $(:,:, 3)$;

Red, green, and blue signals are used in CRT displays. Because of mutual redundancy in these signal, for storage and transmission purpose they are not efficient [7].

\subsubsection{YUV color spaces}

YUV color space is used in PAL (Phase Alternating Line) format in television broadcasting system. RGB color spaces can be transformed to YUV color space using equations (5), (6) and (7) and YUV color space can be transformed back to RBG color space using equations(8), (9) and (10).

$$
\begin{aligned}
& \mathrm{Y}=0.299 * \mathrm{R}+0.587 * \mathrm{G}+0.114 * \mathrm{~B} ; \\
& \mathrm{U}=-0.147 * \mathrm{R}-0.289 * \mathrm{G}+0.436 * \mathrm{~B} ; \\
& \mathrm{V}=0.615 * \mathrm{R}-0.515 * \mathrm{G}-0.100 * \mathrm{~B} ; \\
& \mathrm{R}=\mathrm{Y}+1.139834576 * \mathrm{~V} ; \\
& \mathrm{G}=\mathrm{Y}-.3946460533 * \mathrm{U}-.58060 * \mathrm{~V} ; \\
& \mathrm{B}=\mathrm{Y}+2.032111938 * \mathrm{U} ;
\end{aligned}
$$

\subsubsection{YIQ Color Space}

YIQ color space is defined by NTSC (National Television Systems Committee) used in television broadcasting in US. It consist of three components: the $\mathrm{Y}$ component i.e. luminance represents the grayscale information while I and Q components represents color information i.e. chrominance. RGB color space can be mapped to YIQ using equations (11), (12) and (13) and YIQ color space can be mapped to RGB using equations (14), (15) and (16).

$$
\begin{aligned}
& \mathrm{Y}=0.299 * \mathrm{R}+0.587 * \mathrm{G}+0.114 * \mathrm{~B} \\
& \mathrm{I}=-0.596 * \mathrm{R}-0.274 * \mathrm{G}+0.322 * \mathrm{~B} \\
& \mathrm{Q}=0.211 * \mathrm{R}-0.522 * \mathrm{G}-0.311 * \mathrm{~B} \\
& \mathrm{R}=\mathrm{Y}+0.956 * \mathrm{I}+0.621 * \mathrm{Q} ; \\
& \mathrm{G}=\mathrm{Y}-0.272 * \mathrm{I}-0.647 * \mathrm{Q} ; \\
& \mathrm{B}=\mathrm{Y}+1.106 * \mathrm{I}+1.702 * \mathrm{Q} ;
\end{aligned}
$$

\subsubsection{YCbCr Color space}

$\mathrm{YCbCr}$ color space is used in digital video. $\mathrm{Y}$ represents the luminance information and the difference between the blue component and reference value is stored in $\mathrm{Cb}$ and the difference between red component and reference value is stored in $\mathrm{Cr}$.

\section{THE PROPOSED METHOD}

The Proposed technique is executed in all YUV, YIQ and $\mathrm{YCbCr}$ color spaces by implementing secured robust algorithm. Following algorithm is applied for Y, U, V, I, Q, $\mathrm{Cb}$ and $\mathrm{Cr}$ components one by one according to different color spaces.

\subsection{Watermark Embedding Process}

Step 1: Read the Color cover image and apply 2D-LWT on cover image.

[ca,ch,cv,cd]=lwt2(im2double(rgbimage1),'haar');

Step 2: Extract the Y, U and V component using R, G and $\mathrm{B}$ component from ch sub-band as per equations (5), (6) and (7)

Step 3: Apply SVD to Y component.

[U_imgb1, S_imgb1, V_imgb1]= svd(Y);

Step 4: Read the watermark image. Scramble the watermark by Fibonacci-Lucas transform say sw image using equation (19).

Step 5: Embed the watermark using scaling factor to singular values extracted in step 3 .

temp=S_imgb1+(i*swimage $)$

[U_wimgb S_wimgb V_wimgb]=svd(temp);

Step 6: Apply inverse SVD to find watermarked Y component.

wimgY = U_imgb1*S_wimgb*V_imgb1';

Step 7: convert wimgY component, $\mathrm{U}$ and $\mathrm{V}$ to $\mathrm{R}, \mathrm{G}$ and $\mathrm{B}$ component using equation (8), (9) and (10) to get recovered_ch sub-band in RGB color space.

Step 8: Apply inverse 2D-LWT to get the final watermarked image.

\subsection{Watermark Extracting Process}

Step 1: Read the watermarked image and apply 2D-LWT to watermarked image.

[wm_ca,wm_ch,wm_cv,wm_cd]=lwt2(rgbimage,'haar');

Step 2: Extract the Y, U and V component using R, G and B component from wm_ch sub-band as per equation (5), (6) and (7).

Step 3: Apply SVD to Y3 component. [U_imgb3,S_imgb3,V_imgb3]= svd(Y3);

Step 4: Apply following formula to extract the scrambled recovered watermark.

$\mathrm{SN}=\mathrm{U} \_$wimgb $* \mathrm{~S} \_$imgb3*V_wimgb';

$\operatorname{simg}=\left(\mathrm{SN}-\mathrm{S} \_i m g b 1\right) / \mathrm{i}$;

Step 5: Apply inverse Fibonacci-Lucas Transform to recover the final watermark. 


\section{EXPERIMENTAL RESULT}

The proposed technique is designed for maintaining the perceptual transparency and Robustness of the watermarked image.

Perceptual quality matrix means by embedding watermark to the cover image, the perceived quality of the cover image should not be degraded. For the comparison of the degraded quality MSE is the measure of fidelity.

$\operatorname{MSE}(X, Y)=\frac{1}{M x N} \sum_{i=0}^{M} \sum_{j=0}^{N}\left(X_{i, j}-Y_{i, j}\right)^{2}$

Peak Signal to Noise ratio (PSNR) between original cover image and watermarked image can be found using MSE:

$P S N R=10 \log _{10} \frac{255^{2}}{M S E}$

To measure the robustness of the watermarking technique Normalized Correlation (NC) can be used. The NC i.e. the correlation between original watermark and extracted watermark can be formulated as:

$$
N C(X, Y)=\frac{\sum_{N=0}^{N-1} \sum_{M=0}^{M-1} X[N, M] . Y[N, M]}{\sqrt{\sum_{N=0}^{N-1} \sum_{M=0}^{M-1} X^{2}[N, M] \sum_{N=0}^{N-1} \sum_{M=0}^{M-1} Y^{2}[N, M]}}
$$

This Proposed scheme is implemented in Mat lab. Many tests are carried out for comparative analysis of all the channels in YUV, YIQ and YCbCr color spaces. The scaling factor is varied from 0.05 to 1.85 with the increment of 0.2. The PSNR and NC values are noted for all the channels in YUV, YIQ and $\mathrm{YCbCr}$ color spaces as shown in Fig. 3 and Fig. 4. Maximum recorded PSNR for $\mathrm{V}$ channel is 90.6039 and upto 65.5971 for $\mathrm{Y}$ channel in $\mathrm{YCbCr}$ color space. Maximum NC is recorded in $\mathrm{Y}$ channel for all the color spaces.

Applying various attacks like Average filter, Gaussian filter, Gaussian Noise, Salt \& pepper noise, Blur, Motion Blur, Crop, JPEG Compress, Rotate, Sharpening, the proposed technique is tested for all the channels of the different color spaces is shown in the Fig. 6.

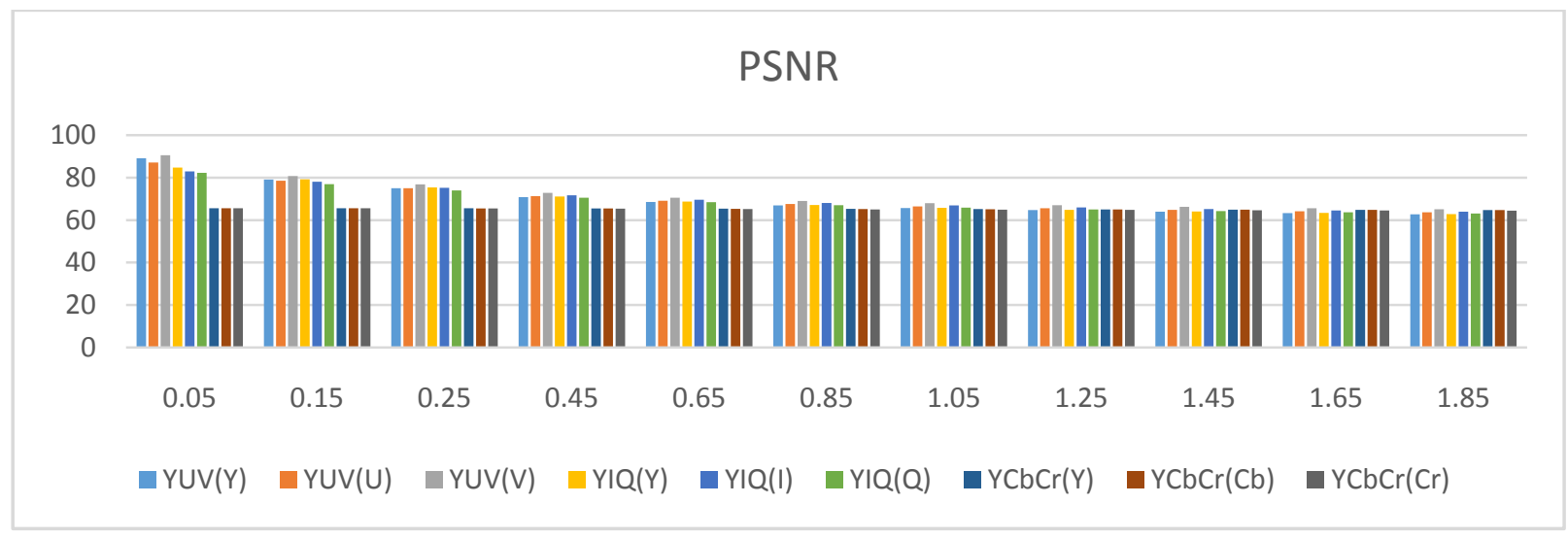

Fig 3: Comparative Imperceptibility in terms of PSNR for different channel of YUV, YIQ and YCbCr color spaces for different scaling factor.

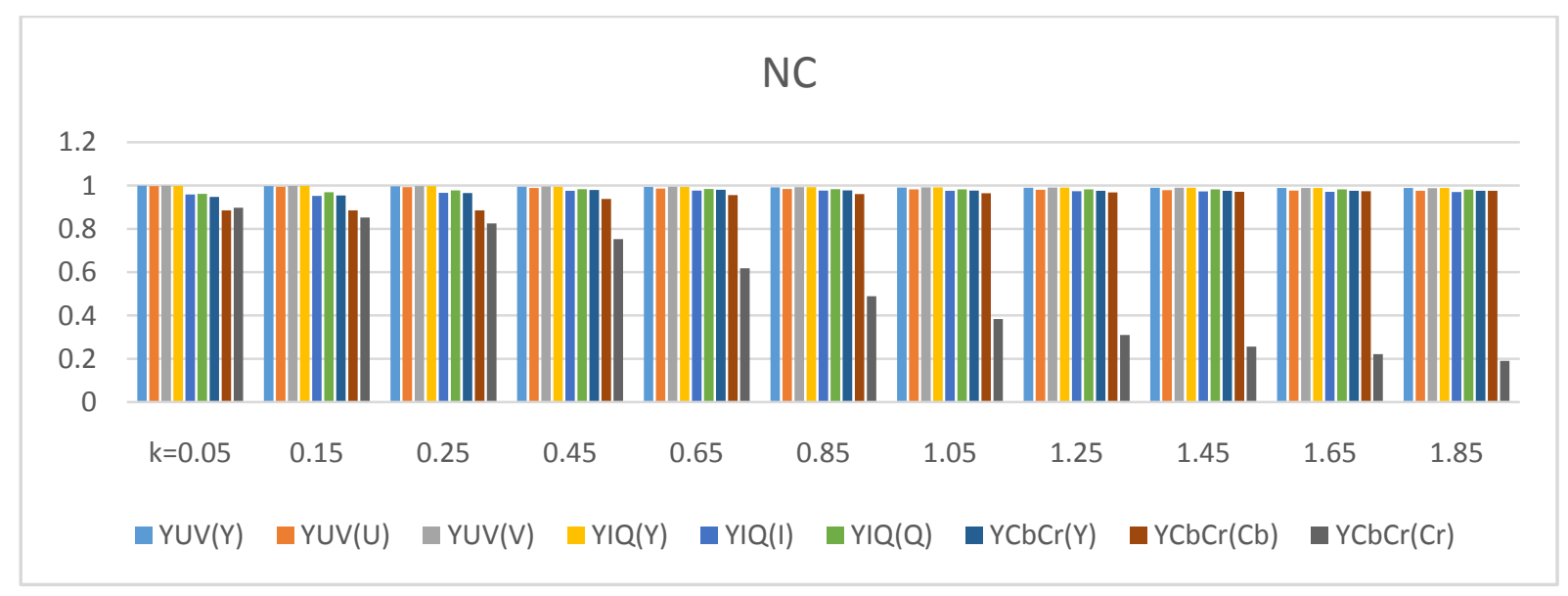

Fig 4: Comparative NC values for different channel of YUV, YIQ and YCbCr color spaces for different scaling factor.

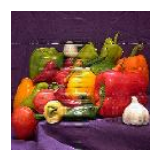

YUV - Y channel

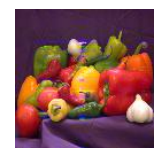

YUV - U channel

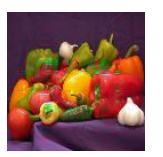

YUV - V channel

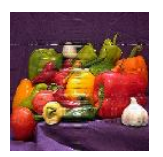

YIQ - Y channel
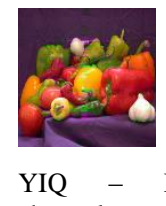
channel

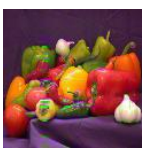

YIQ - Q channel

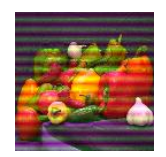

$\mathrm{YCbCr}-\mathrm{Y}$ channel

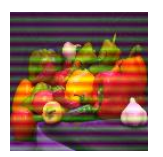

$\mathrm{YCbCr}-\mathrm{Cb}$ channel

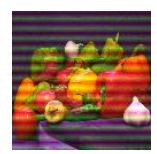

$\mathrm{YCbCr}-\mathrm{Cr}$ channel 


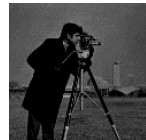

YUV - Y channel

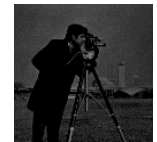

YUV - U channel

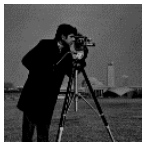

YUV - V channel
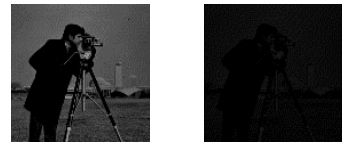

YIQ - Y YIQ channel

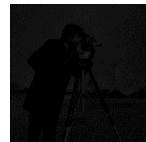

YIQ

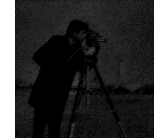

YIQ - Q $\mathrm{YCbCr}-\mathrm{Y}$ channel

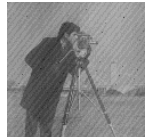

$\mathrm{YCbCr}-\mathrm{Cb}$ channel

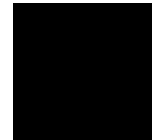

$\mathrm{YCbCr}-\mathrm{Cr}$ channel

Fig 5: Watermarked image and Extracted watermark from YUV, YIQ and YCbCr color spaces for scaling factor 0.85

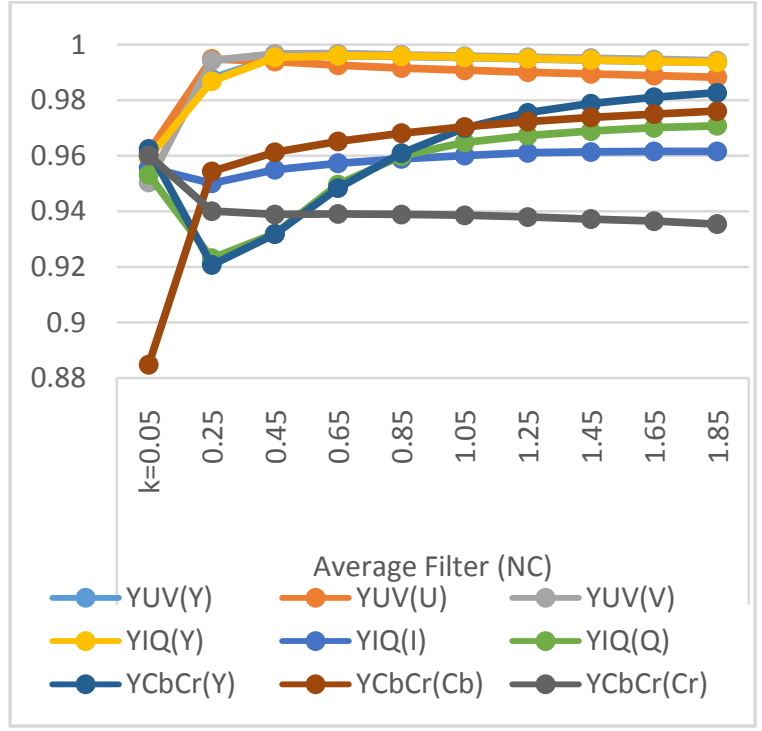

(A)

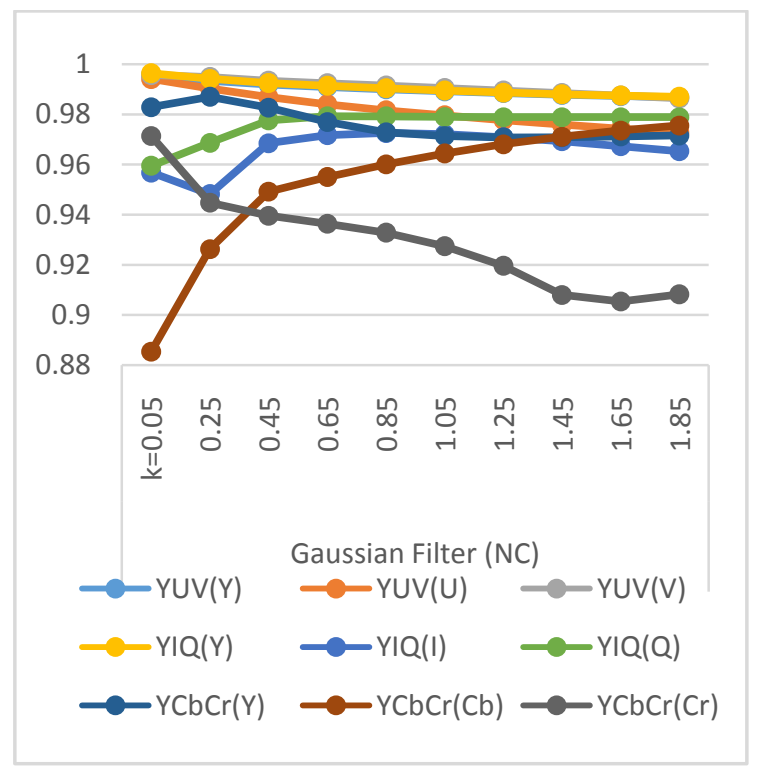

(C)

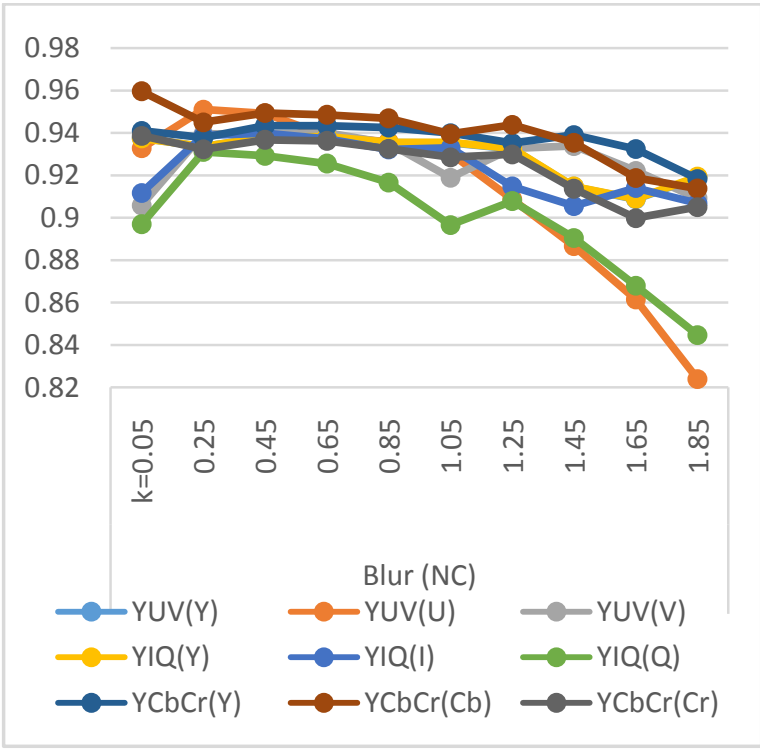

(B)

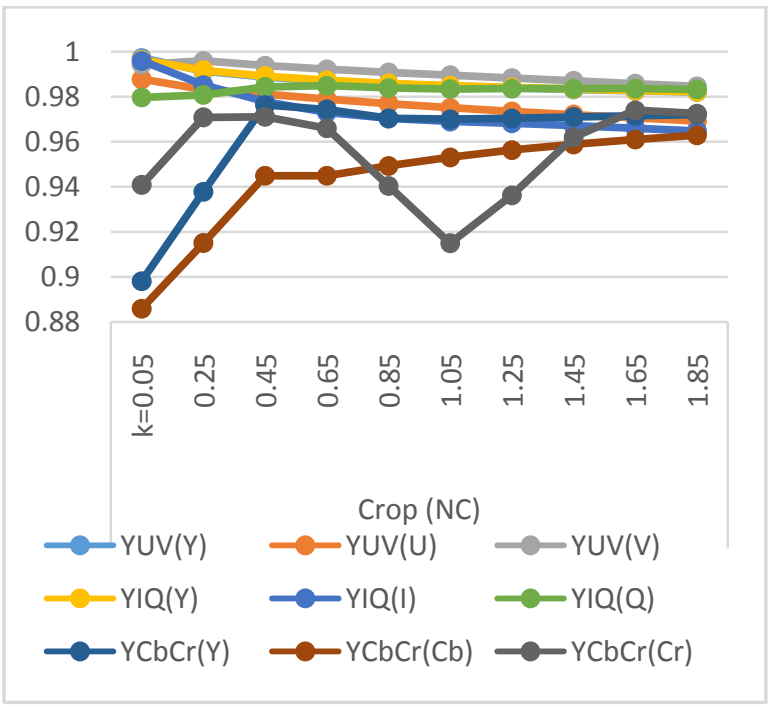

(D) 

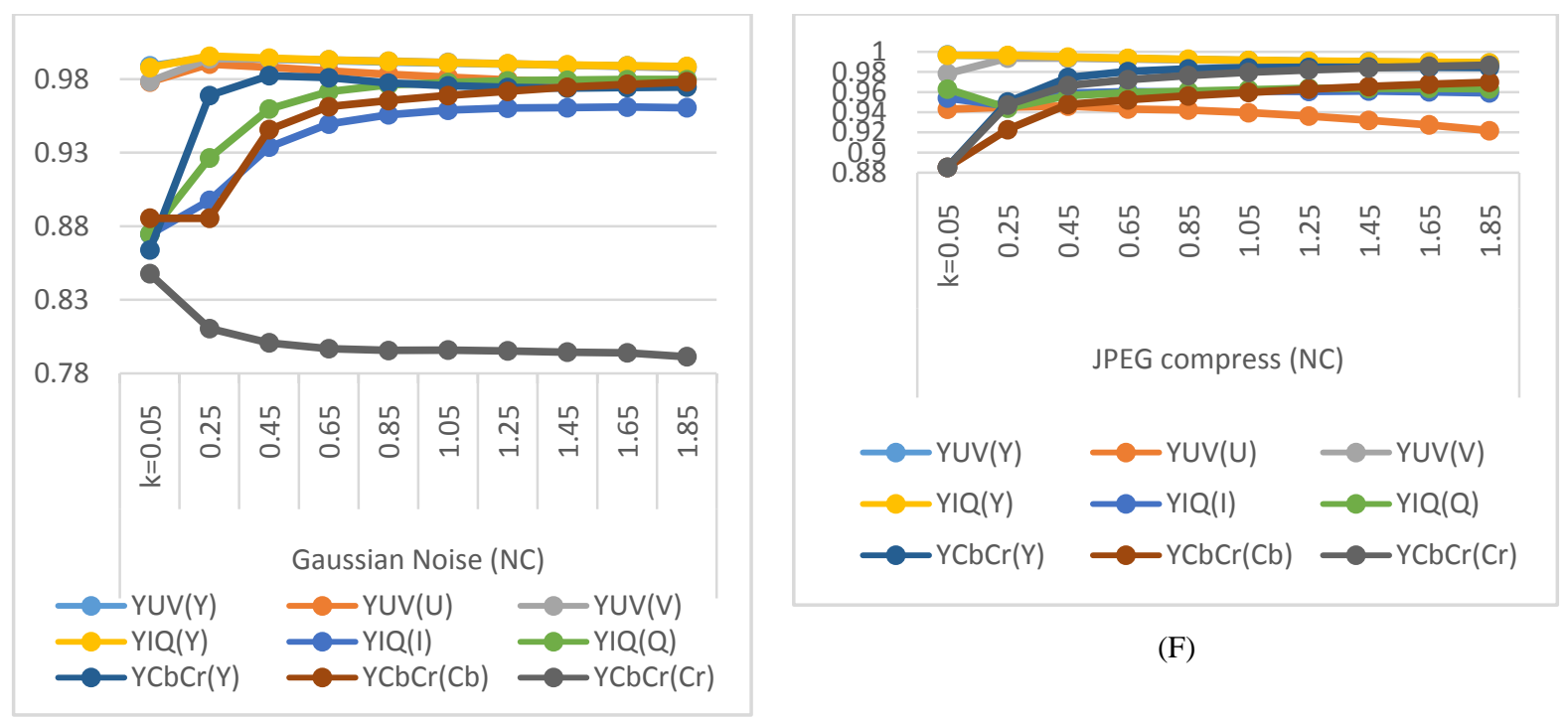

(F)

(E)
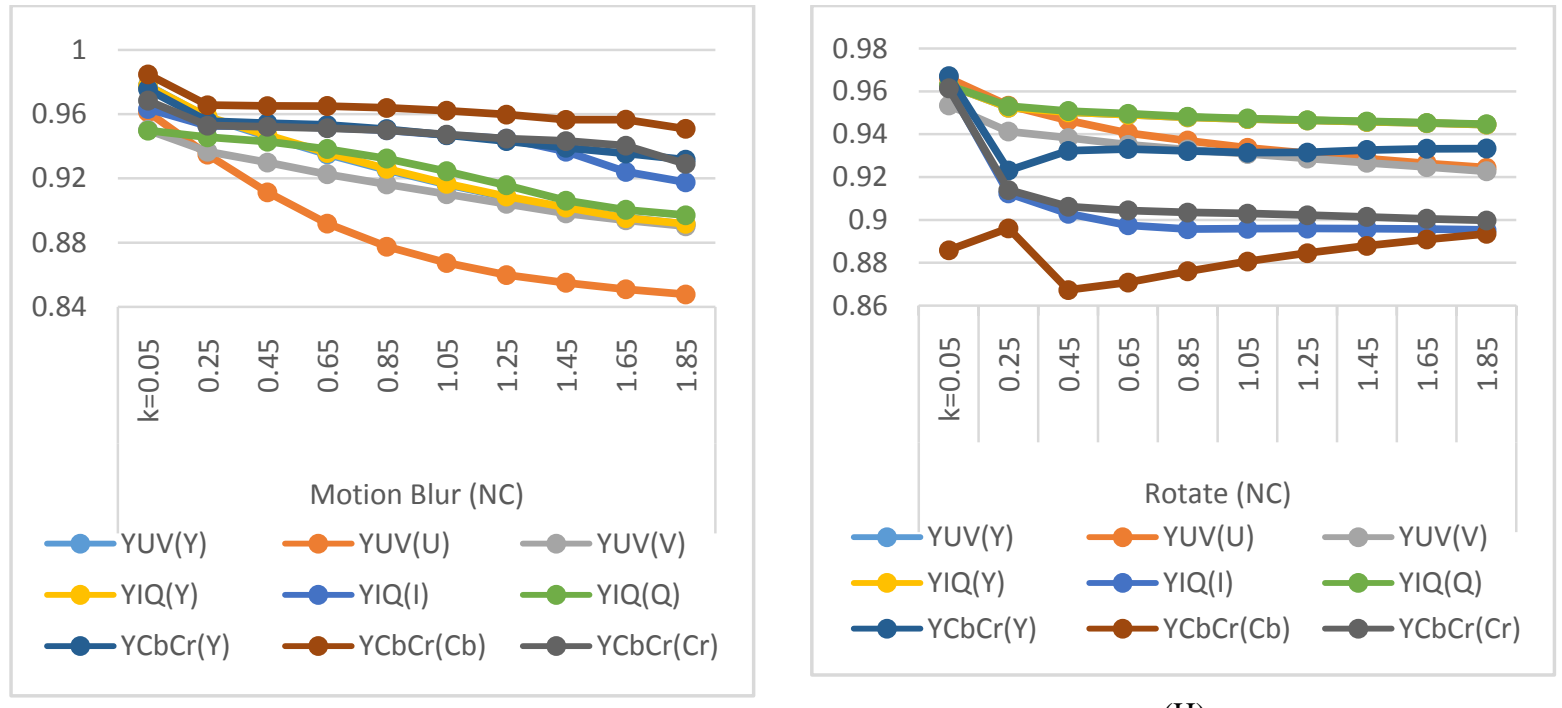

(G)

(H)

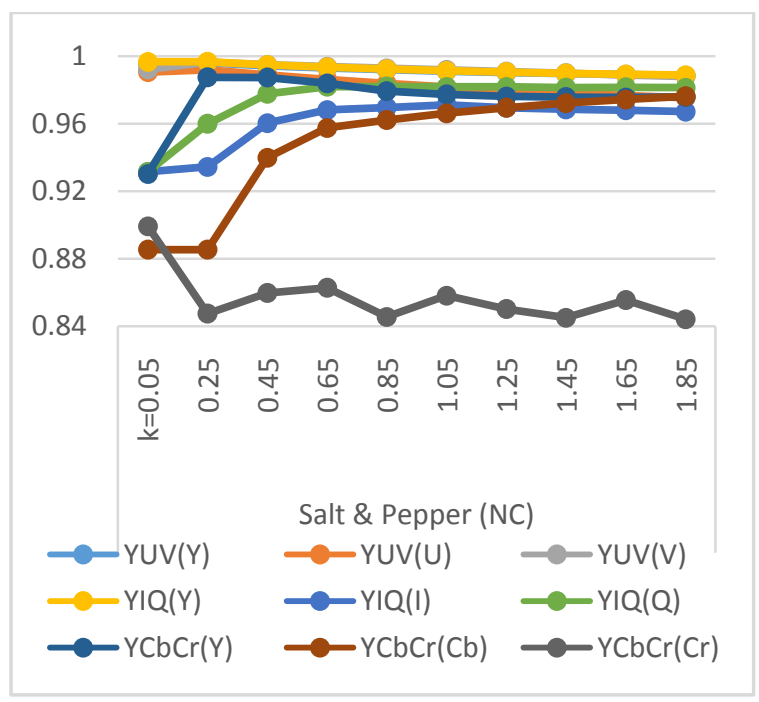

(I)

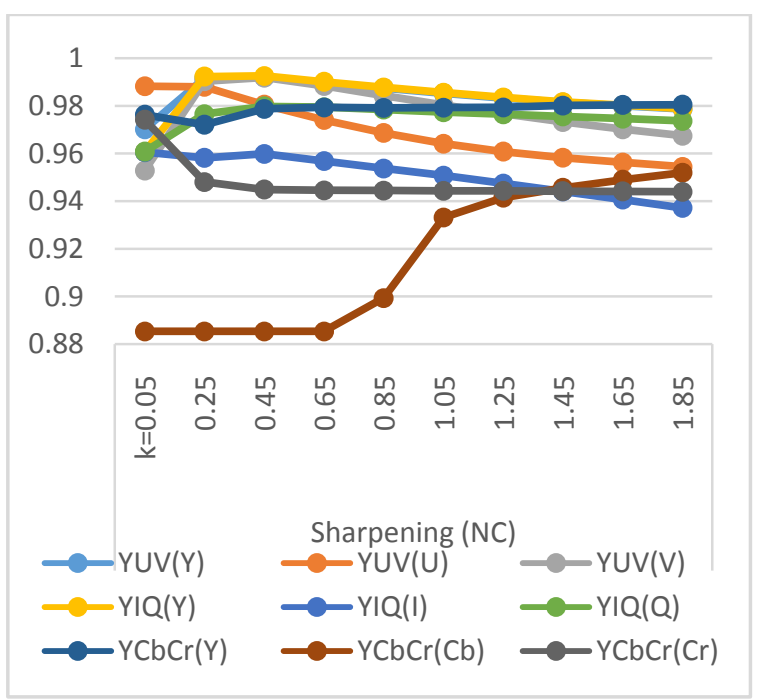

(J)

Fig 6: Comparative Robustness in terms of NC for different channel of YUV, YIQ and YCbCr color spaces for different scaling factor. 


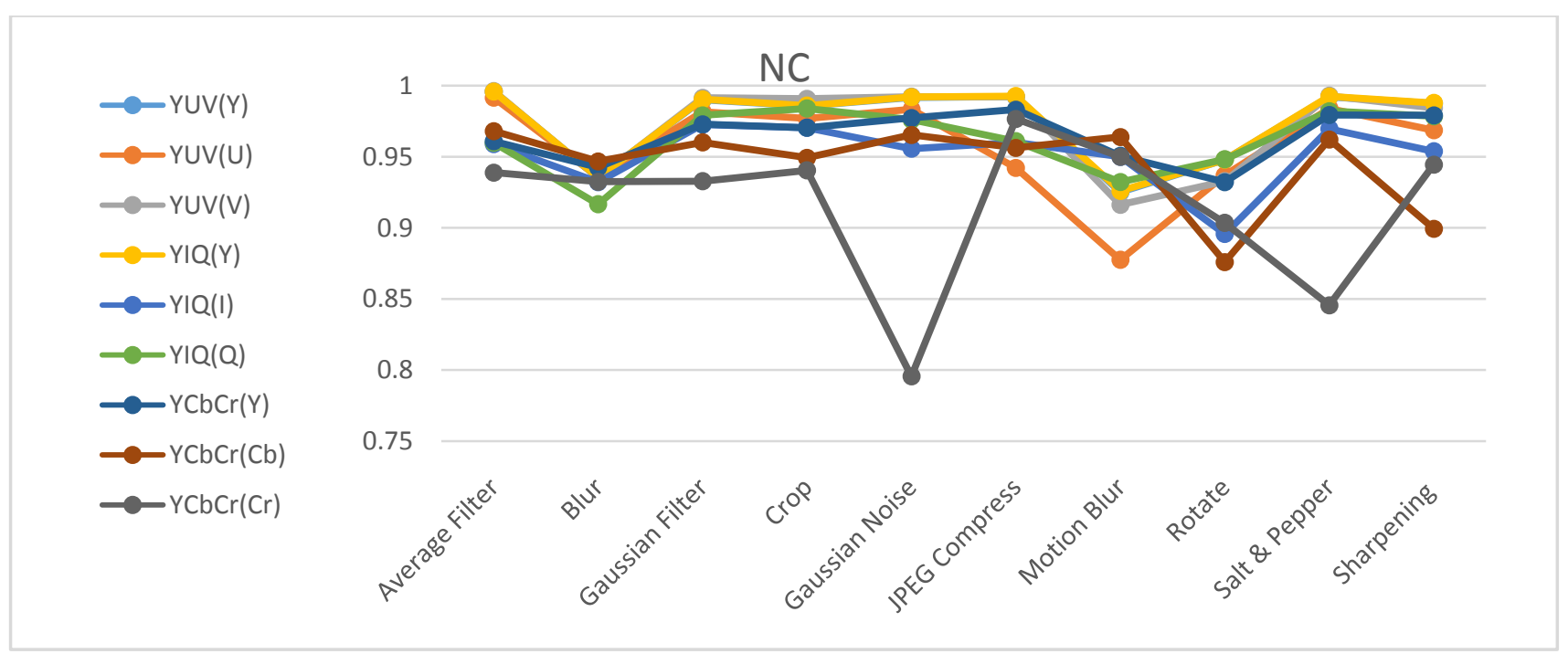

Fig 7: Comparative Robustness performance in terms of NC for different channel of YUV, YIQ and YCbCr color spaces for scaling facto $r=0.85$.

From the Fig. 7 it is observed that embedding watermark in $\mathrm{V}$ channel of YUV color space, Y channel of YIQ color space and $\mathrm{Y}$ channel of $\mathrm{YCbCr}$ color space is more robust.

\section{CONCLUSION}

In this paper Fibonacci-Lucas Transform scrambling algorithm is used for security purpose. YUV, YIQ and $\mathrm{YCbCr}$ color spaces are evaluated by implementing this proposed secured, robust algorithm. It is found that watermark embedding in LWT domain gives better perceptual quality and robustness compared to DWT domain. Maximum recorded PSNR for V channel is 90.6039 and up to 65.5971 for $\mathrm{Y}$ channel in $\mathrm{YCbCr}$ color space. Maximum $\mathrm{NC}$ is recorded in $\mathrm{Y}$ channel for all the color spaces. Embedding the gray-scale watermark in $\mathrm{V}$ channel of YUV color space, $\mathrm{Y}$ channel of YIQ color space and Y channel of YCbCr color space is more robust. This technique can be extended for different color spaces like HSV, HSL, CMYK, etc. This technique can be extended for video watermarking also.

\section{REFERENCES}

[1] Hongqin Shi, Fangliang Lv, Yiqin Cao, "A Dual Color Image Watermarking Scheme Based on Non-overlapping Blocks with Circulation", Journal of Computers, vol. 9, no. 8 , august 2014 .

[2] P.Ramana Reddy, Munaga .V.N.K.Prasad, D. Sreenivasa Rao, "Robust Digital Watermarking of Color Images under Noise attacks", International Journal of Recent Trends in Engineering, Vol 1, No. 1, May 2009.

[3] Baisa L. Gunjal, "Wavelet Based Color Image Watermarking Scheme Giving High Robustness And Exact Corelation", International Journal Of Emerging Trends In Engineering And Technology (Ijetet) Vol. I No. 1, 2011.

[4] D.Vaishnavi and T.S.Subashini, Robust and Invisible Image Watermarking in RGB Color space using SVD, International Conference on Information and Communication Technologies (ICICT 2014), Procedia Computer Science 46 (2015) 1770 - 1777.

[5] N.Venkatram, L.S.S.Reddy, P.V.V.Kishore, Blind Medical Image Watermarking with LWT - SVD for TelemedicineApplications, WSEAS TRANSACTIONS on SIGNAL PROCESSING, Volume 10, 2014.

[6] Poulami Ghosh, Rilok Ghosh, Souptik Sinha, Aruna Chakroborty 2012. "A Novel Digital Watermarking Technique for Video Copyright Protection" CS \& IT 06, pp. 601-609.

[7] P. Sathya and L. Malathi, "Classification and Segmentation in Satellite Imagery Using Back Propagation Algorithm of ANN and K-Means Algorithm", International Journal of Machine Learning and Computing, Vol. 1, No. 4, October 2011 